\title{
LIBRARY AUTOMATION SOFTWARE: A COMPARATIVE STUDY OF KOHA, LIBSYS, NEWGENLIB AND SOUL
}

\author{
UMESHA NAIK \\ Assistant Professor, Department of Library and information Science, \\ Mangalore University, Mangalore, Karnataka, India
}

\begin{abstract}
Libraries and information centers have planned library automation efforts and practices, in order to provide their best services to the right users, in the right time, at the mode. A process of great change has been taking place in libraries, due to the impact of information technology and application of computers, in library work. In this study, the investigator considering a Comparative study of KOHA. LibSys, New Gen Lib and SOUL both two Open Sources Software and two proprietary Integrated library management software. This study also focuses on the general and specific features, of the four most popular integrated library management software. A brief dissection was done on popular software package, which are available for automation purposes. This article also focused on the overview of library automation software packages, and their features and functions of library management.

KEYWORDS: Library Automation, KOHA, LibSys, NewGenLib, SOUL, ILMS, Integrated Library Management Software, Computerisation, Library Management
\end{abstract}

Received: Oct 10, 2016; Accepted: Nov 26, 2016; Published: Dec 13, 2016; Paper Id.: IJLSRDEC20168

\section{INTRODUCTION}

Library automation is the process, which uses computers, to automate the typical procedures of libraries, such as cataloging and circulation. Today, a number of application software is available, either free/open source software or commercial with distinct feature. Library automation, impacts the five laws of library science, in providing the right information to the right users, right way, at the right time. Software selection depends on the financial management support, and the Open Source Initiative (OSI) is a good opportunity, to select and implement without any financial commitment; it reduces the cost of the library automation.

Library automation is the process of using the ICT tools, for easy working and saving the human power and time. Library automation is the general term, for information and communications technologies (ICT), that are used to replace manual systems in the library

\section{LIBRARY AUTOMATION SOFTWARE}

Library automation software is very useful for the maintenance of easy access, use and process. In the software part, the open source software compares very conveniently, than the free and commercial software. Library automation supports the mechanization of the traditional library operations modules, like acquisition, serial control, cataloguing and circulation control, OPAC, Budgeting, reporting, etc. The objective of this paper 
is to give an overview of the factors considered, and the checklist for selection and evaluation of library automation. It helps the library and information professionals, to find more time for the vital functions of the information provision. The major function, library automation software should provide are: acquired, cataloguing, serial control, circulation control, information processing and retrieval, OPAC, report generation, documentation etc.

\section{OBJECTIVES}

The following objectives are of this work;

- To know more about the four Integrated Library Management Software for Libraries

- To understand the functionality and technology used in KOHA, LibSys, NewGenLib and SOUL.

- To explore the special features and standards, maintain in this software

- $\quad$ To know the basic and advance modules of this software

- To find out the popularity, overall status and get a clear-cut idea which software is better for automated libraries.

\section{METHODOLOGY}

The four popular library automation software, has been included in this study. The features, standards and functions of these four software detailed data, were retrieved from the internet. The methodology is a content analysis of the websites of the Koha, LibSys NewGenLib and SOUL, only. All the four software were more powerful and offered by some other companies, but this study has focused on categories like, including general information about products, service, status, and use. The content analysis work was done, during January to November, 2016. The following are the list of electronic information resource gateways, selected for the study:

\section{SCOPE AND LIMITATION OF THE STUDY}

There are a number of proprietary and open source software, for library automation and management available worldwide, in this study, the investigator selected two proprietary and two open source software. The investigator highlighted only the main modules, functions features and standards of these four popular library automation, and management software.

\section{Koha}

Koha, is the first free software library automation package. Koha is distributed under the Free Software General Public License (GPL), version 3 or later. Worldwide, almost all sizes of library and information centers use the Koha software for their day to day library works. It notices, offline is a true enterprise-class ILS, with comprehensive functionality including basic and advanced options. The Koha software includes the main modules for acquisitions, circulation, cataloging, serials management, authorities, flexible reporting, label printing, multi-format notices, offline circulation for when, Internet access is not available, and much more. Koha will work for library consortia of all sizes, multi-branch, and single-branch libraries. Koha will support for a large number of available languages, with more languages in everyyear. The powerful searching tools including full text searching and enhanced catalogue display, that can use content from Amazon, Google, Library Thing, Open Library, and Syndetics, among others.

The Koha software contains the major library standards and protocols, such as MARC 21, UNIMARC, z39. 50, 
SRU/SW, SIP2, SIP/NCIP etc. It ensures interoperability between Koha and other systems and technologies, while supporting existing workflows and tools. The main modules of OPAC, circulation and self-checkout and some other module interfaces are based on standards-compliant WWWW technologies - XHTML, CSS and Javascript-making.

\section{Major Features and Functionalities}

KOHA have more features compared to other ILMS; the main features and functions of KOHA are;

- The entire software has a web enabled interface and Web 2.0 facilities like tagging, comment, social sharing and RSS feeds etc.

- The Union catalog facility and customizable search facilities

- Circulation and borrower management, including RFID features

- The major acquisition system, including budgets and pricing information (including supplier and currency conversion) and simple acquisition system for the smaller library

- The main ability, to cope with any number of branches, patrons, patron categories, item categories, items, currencies and other data, etc.

- Serials control for magazines or newspapers and other periodical functions

- Almost all the sections, the reporting facilities and reading lists for members, and other details

- Both off-line and on- line circulation facilities

- Segmentation of the line and it will support any independent operating system. Linux, Unix, Mac.

- The library Standards and protocols, Full MARC21 and UNIMARC support for professional cataloguing, Z39.50 server, Export and import records, ISO2709 and major open source database type (text, RDBMS), SQL, MYSQL.

- The multilingual and multi-user support

- Full featured catalogue, circulation, acquisitions, Serial management module, library, stock management, online reservation and web based OPAC, public to search the catalogue and

- It supports for print the barcode.

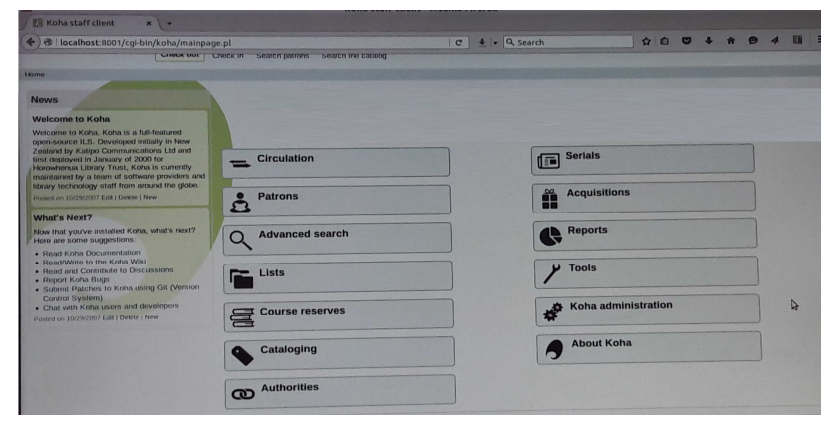

Figure 1: Koha Main Menu 


\section{Libsys}

The Lib 2.0 based interface LibSys, delivers unparalleled satisfaction for both patrons and library staff. LibSys gives end to end manageability of the library operations, through its complete modules. The web based interface provides the functions and solution, it also provides platform independence. The main features in the software are most advanced OPAC, makes LibSys most rewarding choice, for all the Librarians. The value added features and functions, adopted in this interface and it is one more web enabled software, for LIS professional.

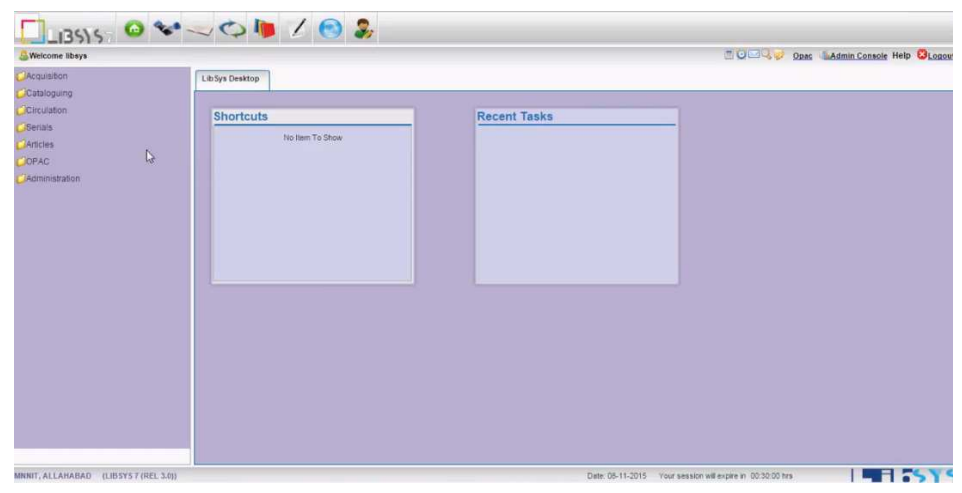

Figure 2: Libsys Main Menu

\section{Major Features and Functionalities}

- Google Web Toolkit based Graphical User Interface, with multitasking feature

- This software supports Unicode, federated searching with customizable look \& feel and user notification through E-mail and SMS

- $\quad$ This interface also supports RSS feeds and integration with Google Books, Book Finder, etc.

- The interactive features like online reviews, ratings, renewals, reservations, etc., to deliver patron satisfaction.

- The international standards compliance - MARC21; Unicode; SRU/SRW; Z39.50; NCIP (NISO); SICI Barcode is adopted in this tool.

- The major modules like acquisition; cataloguing; circulation; serials management; article indexing; web OPAC; customizable reports and other inbuilt functions.

\section{Newgenlib}

The NewGenLib, is an integrated library management system, developed by Verus Solutions Pvt Ltd. Kesavan Institute of Information and Knowledge Management in Hyderabad, is supporting and providing the domain and expertise. The first version 1.0 was released in the year 2005. On 9 January 2008, it was declared Open Source Software, under the GNU GPL, for LIS professionals. 


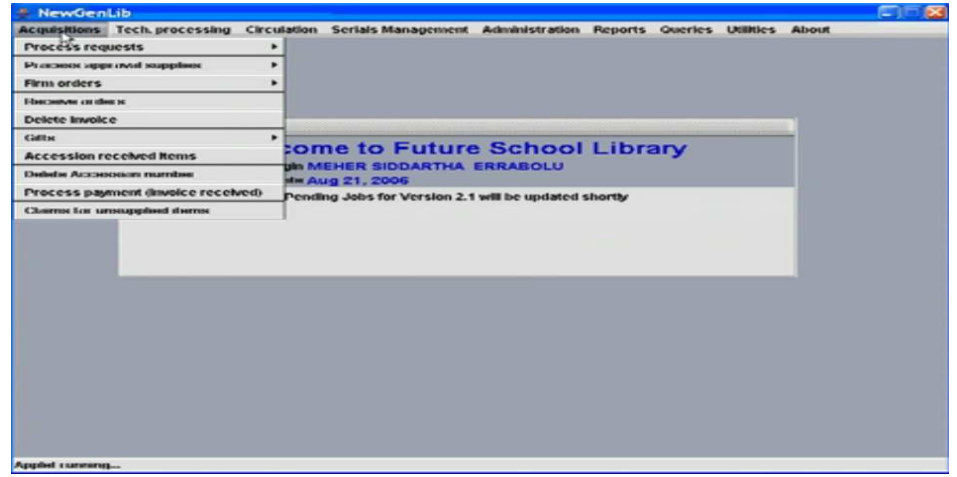

Figure 3: Newgenlib Main Menu

\section{Major Features and Functionalities}

- $\quad$ The web enabled interface supports and uses Java Web Start ${ }^{\mathrm{TM}}$ Technology

- The compatibility and standards like complies with international metadata, and interoperability standards: MARC21, MARC-XML, z39. 50, SRU/W, OAI-PMH, etc.

- Scalable, manageable, efficient and one of the uses chiefly open source components

- The independent operating systems like Windows and Linux flavors available

- The z39. 50 client for federated searching and supports multi-user and multiple security levels

- $\quad$ The internationalized application $(\mathrm{I} 18 \mathrm{~N})$ like

- Unicode 4.0 complaint

- $\quad$ Easily extensible to support other languages

- Data entry, storage, retrieval in any (Unicode 3.0) language

- $\quad$ The networking features - Hierarchical and Distributed networks

- Automated email/instant messaging integrated into different functions of the software and it will support for RFID integration, and use XML-based Open Office templates

- The extensive use of set up parameters, enabling easy configuration of the software to suit specific needs.

- It allows digital attachments to metadata

Soul

Software for University Libraries (SOUL), was developed by INFLIBNET Centre. It is a state-of-the-art Integrated Library Management System (ILMS), designed and developed, based on requirements of college and university libraries. One of the user-friendly software, developed to work under client-server environment. This software supports the international standards for bibliographic formats, networking and circulation protocols. The well known LIS and CS experts, spent their valuable time for developing this software, and based on the comprehensive study, discussions and deliberations with the senior professionals of the country; the software was designed to automate all housekeeping operations, in the library. The first version SOUL 1.0 was released during CALIBER 2000. The latest version of the 
software is SOUL 2.0; it was released in January 2009. The new version of SOUL 2.0 is designed for the latest versions of MS-SQL and MySQL (or any other popular RDBMS). SOUL 2.0 is the support for international standards, such as MARC 21 bibliographic format, Unicode based Universal Character Sets for multilingual bibliographic records, and NCIP 2.0 and SIP 2 based protocols, for electronic surveillance and control

\section{Major Features and Functionalities}

- The multilingual support for Indian and foreign languages based on UNICODE

- The international standards Compliant to International Standards such as MARC21, AACR-2, MARCXML, NCIP 2.0 protocol for RFID and other related applications, especially for electronic surveillance and self checkout \& check-in;

- The client-server based architecture, user-friendly interface supports multi-platform, for bibliographic database such as MySQL, MS-SQL or any other RDBMS;

- It supports cataloguing of e-resources, such as e-journals, e-books, virtually any type of material;

- The latest version of SOUL 2.0, supports access the digital library and facilitate links to full-text articles and other digital objects;

- The online copy cataloguing from MARC21 supported bibliographic database, and it also provides default templates for data entry of different type of documents. Based on customization, the user can also customize their own data entry templates, for different type of documents;

- It provides freedom to users, for generating reports of their choice and format, along with the template and query parameters, it provides facility to send reports through e-mail, allows users to save the reports in various formats such as Word, PDF, Excel, MARCXML, etc.;

- The highly versatile and user-friendly OPAC, with simple and advanced search modules supports the users, to export their search results into PDF, MS Excel, and MARCXML format;

- It supports the authority files like personal names, corporate body, subject headings and series name, and also supports data exchange through ISO-2709 standard;

- It provides simple budgeting system and single window operation, for all major circulation functions;

- The region-wise support for maintenance, through regional coordinators and online and offline support by email, chat and through a dedicated telephone line, during office hours for maintaining the status of work. 


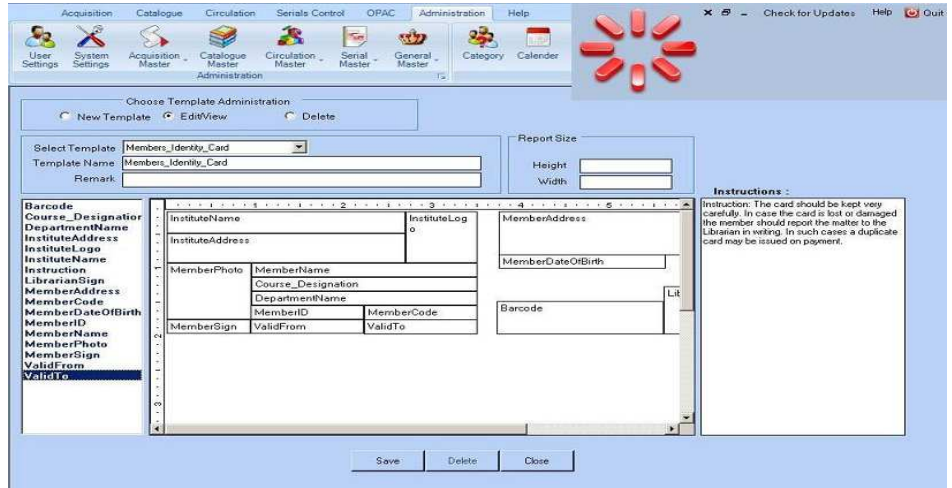

Figure 4: Soul Main Menu

\section{Analysis of Data}

To assess the status of information, these software packages and the investigator, access the website (URL) and collect the necessary data in this regard, from the site. Table 1, provides overall features and functions of library automation software, out of four library management software. The selected four software modules, features and functions are highlighted in this study. Further, this paper discusses the need for evaluation of the library automation software, factors to be considered, checklist used for selection and evaluation of library automation software.

Table 1: Features and Functions of ILMS Tools

\begin{tabular}{|c|c|c|c|c|}
\hline Features & КОНА & LibSys & NewGenLib & SOUL \\
\hline Alert Service & $\sqrt{ }$ & $\sqrt{ }$ & $\sqrt{ }$ & $\sqrt{ }$ \\
\hline Authority Files & $\sqrt{ }$ & $\sqrt{ }$ & $\sqrt{ }$ & $\sqrt{ }$ \\
\hline Barcode & $\sqrt{ }$ & $\sqrt{ }$ & $\sqrt{ }$ & $\sqrt{ }$ \\
\hline Biometrics & $\sqrt{ }$ & $\sqrt{ }$ & $X$ & $\mathrm{X}$ \\
\hline Copy Cataloguing & $\sqrt{ }$ & $\sqrt{ }$ & No & $\sqrt{ }$ \\
\hline Cost (full Features & Free/OSS & $10 \mathrm{Lakh}+$ & Free/OSS & 80,000 \\
\hline Developers & $\begin{array}{c}\text { Koha } \\
\text { Community }\end{array}$ & LIBSYS Ltd & $\begin{array}{l}\text { Verus Solutions } \\
\text { Pvt Ltd. }\end{array}$ & $\begin{array}{l}\text { INFLIBNET } \\
\text { Centre }\end{array}$ \\
\hline Digital Resources & $\sqrt{ }$ & $\sqrt{ }$ & $\sqrt{ }$ & $\sqrt{ }$ \\
\hline Effectiveness & $\sqrt{ }$ & $\sqrt{ }$ & $\sqrt{ }$ & $\sqrt{ }$ \\
\hline Expandability & $\sqrt{ }$ & $\sqrt{ }$ & $\sqrt{ }$ & $\sqrt{ }$ \\
\hline Federated Searching & $\sqrt{ }$ & $\sqrt{ }$ & $\sqrt{ }$ & $\sqrt{ }$ \\
\hline Flexibility & $\sqrt{ }$ & $\sqrt{ }$ & $\sqrt{ }$ & $\sqrt{ }$ \\
\hline Free User Manual & $\sqrt{ }$ & $\mathrm{X}$ & $\sqrt{ }$ & $X$ \\
\hline Google Web Toolkit & $\sqrt{ }$ & $\sqrt{ }$ & $\mathrm{X}$ & $\mathrm{X}$ \\
\hline GUI & $\sqrt{ }$ & $\sqrt{ }$ & $\sqrt{ }$ & $\sqrt{ }$ \\
\hline License & GNU & Proprietary & GNU & Proprietary \\
\hline Multimedia Files & $\sqrt{ }$ & $\sqrt{ }$ & $X$ & $\bar{X}$ \\
\hline Multiple platform & Yes & Yes & Yes & Yes \\
\hline Networking & $\sqrt{ }$ & $\sqrt{ }$ & $\sqrt{ }$ & $\sqrt{ }$ \\
\hline On-site Training & $\sqrt{ }$ & $X$ & $\sqrt{ }$ & $\sqrt{ }$ \\
\hline Operating System & $\begin{array}{c}\text { Linux/Window } \\
\mathrm{s}\end{array}$ & Linux/Windows & Linux/Windows & Linux/Windows \\
\hline RDBMS & MySQL & MS-SQL & $\begin{array}{c}\text { MS-SQL/ } \\
\text { MySQL/ } \\
\text { PostgreSQL/ } \\
\text { Firebird, etc. }\end{array}$ & $\begin{array}{c}\text { MS- } \\
\text { SQL/MySQL }\end{array}$ \\
\hline Reliability & $\sqrt{ }$ & $\sqrt{ }$ & $\sqrt{ }$ & $\sqrt{ }$ \\
\hline
\end{tabular}




\begin{tabular}{|c|c|c|c|c|}
\hline \multicolumn{5}{|c|}{ Table 1: Contd., } \\
\hline RFID Support & $\sqrt{ }$ & $\sqrt{ }$ & $\sqrt{ }$ & $\sqrt{ }$ \\
\hline Security & $\sqrt{ }$ & $\sqrt{ }$ & $\sqrt{ }$ & $\sqrt{ }$ \\
\hline Software label & F Koha & $\equiv$ & NewGenLib & \% soll 2.0 \\
\hline Started Year & 2000 & 1984 & 2008 & 2000 \\
\hline Data entry templates & $\sqrt{ }$ & $X$ & $X$ & $\sqrt{ }$ \\
\hline URL & $\begin{array}{l}\text { https://koha- } \\
\text { community.org } \\
/\end{array}$ & $\begin{array}{c}\text { http://www.libsys. } \\
\text { co.in/ }\end{array}$ & $\begin{array}{l}\text { http://www.verus } \\
\text { solutions.biz/ }\end{array}$ & $\begin{array}{l}\text { http://www.inflib } \\
\text { net.ac.in/soul/ }\end{array}$ \\
\hline User friendliness & $\sqrt{ }$ & $\sqrt{ }$ & $\sqrt{ }$ & $\sqrt{ }$ \\
\hline Version as on 2016 & 16.11 & 7 & 3.1 .2 & 2.0 \\
\hline Web Based Interface & Ш $1 B R A R Y$ SYSTEM & $-13 S \mid S$ & NewGenLib & $=$ \\
\hline Web Server & Apache & - & Java & - \\
\hline
\end{tabular}

The Table 2, highlights the standards adopted and maintained by these four software packages. All the vendors provide software manual and detailed information on the web, but, the LibSys don't have the new technologies like cloud systems, which are not available in SOUL and NewGenLib.

Table 2: Standard Available on the ILMS Tools

\begin{tabular}{|c|c|c|c|c|}
\hline Standards & КОНА & LibSys & NewGenLib & SOUL \\
\hline AACR2 & $\sqrt{ }$ & $\sqrt{ }$ & $\sqrt{ }$ & $\sqrt{ }$ \\
\hline Barcode & $\sqrt{ }$ & $\sqrt{ }$ & $\sqrt{ }$ & $\sqrt{ }$ \\
\hline Cloud & $\sqrt{ }$ & $\sqrt{ }$ & $\mathrm{X}$ & $\mathrm{X}$ \\
\hline DCMI & $\sqrt{ }$ & $\sqrt{ }$ & $\sqrt{ }$ & $\sqrt{ }$ \\
\hline Federated Search & $\sqrt{ }$ & $\sqrt{ }$ & $\sqrt{ }$ & $\sqrt{ }$ \\
\hline ISO 2709 & $\sqrt{ }$ & $\sqrt{ }$ & $\sqrt{ }$ & $\sqrt{ }$ \\
\hline LCSH & $\sqrt{ }$ & $\sqrt{ }$ & $\sqrt{ }$ & $\sqrt{ }$ \\
\hline Manual Available & $\sqrt{ }$ & $X$ & $\sqrt{ }$ & $\sqrt{ }$ \\
\hline MARC & $\sqrt{ }$ & $\sqrt{ }$ & $\sqrt{ }$ & $\sqrt{ }$ \\
\hline MARCXML & $\sqrt{ }$ & $\sqrt{ }$ & $\sqrt{ }$ & $\sqrt{ }$ \\
\hline Metadata & $\sqrt{ }$ & $\sqrt{ }$ & $\sqrt{ }$ & $\sqrt{ }$ \\
\hline $\mathrm{NACO}$ & $\sqrt{ }$ & $\sqrt{ }$ & $\sqrt{ }$ & $\sqrt{ }$ \\
\hline NCIP (NISO) & $\begin{array}{l}\text { NCIP 2.0, SIP } \\
2, \text { I18n, L10n }\end{array}$ & $\begin{array}{l}\text { NCIP 2.0, SIP } \\
2, \text { I18n, L10n }\end{array}$ & $\begin{array}{l}\text { NCIP 2.0, SIP 2, } \\
\text { I18n, L10n }\end{array}$ & $\begin{array}{l}\text { NCIP 2.0, SIP } \\
2, \text { I18n, L10n }\end{array}$ \\
\hline Network & $\sqrt{ }$ & $\sqrt{ }$ & $\sqrt{ }$ & $\sqrt{ }$ \\
\hline OAI-PMH & $\sqrt{ }$ & $\sqrt{ }$ & $\sqrt{ }$ & $\sqrt{ }$ \\
\hline ODBC Support & $\sqrt{ }$ & $\sqrt{ }$ & $\sqrt{ }$ & $\sqrt{ }$ \\
\hline OpenURL & $\sqrt{ }$ & $\sqrt{ }$ & $\sqrt{ }$ & $\sqrt{ }$ \\
\hline RDA & $\sqrt{ }$ & $\sqrt{ }$ & $\sqrt{ }$ & $\sqrt{ }$ \\
\hline Copy Catalogue & $\sqrt{ }$ & $\sqrt{ }$ & $\sqrt{ }$ & $\sqrt{ }$ \\
\hline Report Template & $\sqrt{ }$ & $\sqrt{ }$ & $\sqrt{ }$ & $\sqrt{ }$ \\
\hline RSS & $\sqrt{ }$ & $\sqrt{ }$ & $\sqrt{ }$ & $\sqrt{ }$ \\
\hline Information on the Net & $\sqrt{ }$ & $X$ & $\sqrt{ }$ & $\sqrt{ }$ \\
\hline SRU/SRW & $\sqrt{ }$ & $\sqrt{ }$ & $\sqrt{ }$ & $\sqrt{ }$ \\
\hline State-of-the-art & $\sqrt{ }$ & $\sqrt{ }$ & $\sqrt{ }$ & $\sqrt{ }$ \\
\hline TCP/IP & $\sqrt{ }$ & $\sqrt{ }$ & $\sqrt{ }$ & $\sqrt{ }$ \\
\hline UNICODE & $\sqrt{ }$ & $\sqrt{ }$ & $\sqrt{ }$ & $\sqrt{ }$ \\
\hline Z39.50 & $\sqrt{ }$ & $\sqrt{ }$ & $\sqrt{ }$ & $\sqrt{ }$ \\
\hline Z39.71 & $\sqrt{ }$ & $\sqrt{ }$ & $\sqrt{ }$ & $\sqrt{ }$ \\
\hline
\end{tabular}

Note: Name Authority Cooperative (NACO); Library of Congress Subject Headings (LCSH); Dublin Core 
Metadata Element Scheme (DCMES); Resource Description and Access (RDA); Integrated Library System (ILS); Anglo American Cataloging Rules version Two (AACR2); Open Access Initiative - Protocol for Metadata Harvesting (OAIPMH); MAchine Readable Cataloging (MARC); Transmission Control Protocol/Internet Protocol (TCP/IP); Open Uniform Resource Locator (OpenURL); Search/Retrieve via URL (SRU); Super Robot Wars (SRW); Serial Item and Contribution Identifier (SICI)

The features, functions and other work areas contain the modules of this software. Table 3, focuses on the modules/ menus, available in the four software packages. Almost all the major menu/modules are available in all four software packages, built in KOHA, and in LibSys, the Global Search and Replace features menu is available.

Table 3: Modules Available on ILMS Tools

\begin{tabular}{|c|c|c|c|c|}
\hline Modules & КОНА & LibSys & NewGenLib & SOUL \\
\hline Acquisition & $\sqrt{ }$ & $\sqrt{ }$ & $\sqrt{ }$ & $\sqrt{ }$ \\
\hline Article Indexing & $\sqrt{ }$ & $\sqrt{ }$ & $\sqrt{ }$ & $\sqrt{ }$ \\
\hline Catalogue & $\sqrt{ }$ & $\sqrt{ }$ & $\sqrt{ }$ & $\sqrt{ }$ \\
\hline Circulation & $\sqrt{ }$ & $\sqrt{ }$ & $\sqrt{ }$ & $\sqrt{ }$ \\
\hline Customizable Reports & $\sqrt{ }$ & $\sqrt{ }$ & $\sqrt{ }$ & $\sqrt{ }$ \\
\hline Digital attachments & $\sqrt{ }$ & $\sqrt{ }$ & $\sqrt{ }$ & $\sqrt{ }$ \\
\hline Global Search and Replace & $\sqrt{ }$ & $\sqrt{ }$ & $X$ & $X$ \\
\hline Library administration & $\sqrt{ }$ & $\sqrt{ }$ & $\sqrt{ }$ & $\sqrt{ }$ \\
\hline Multiple security levels & $\sqrt{ }$ & $\sqrt{ }$ & $\sqrt{ }$ & $\sqrt{ }$ \\
\hline Multi-user & $\sqrt{ }$ & $\sqrt{ }$ & $\sqrt{ }$ & $\sqrt{ }$ \\
\hline Reports & $\sqrt{ }$ & $\sqrt{ }$ & $\sqrt{ }$ & $\sqrt{ }$ \\
\hline Serial Control & $\sqrt{ }$ & $\sqrt{ }$ & $\sqrt{ }$ & $\sqrt{ }$ \\
\hline Set up parameters & $\sqrt{ }$ & $\sqrt{ }$ & $\sqrt{ }$ & $\sqrt{ }$ \\
\hline Technical processing & $\sqrt{ }$ & $\sqrt{ }$ & $\sqrt{ }$ & $\sqrt{ }$ \\
\hline Web OPAC & $\sqrt{ }$ & $\sqrt{ }$ & $\sqrt{ }$ & $\sqrt{ }$ \\
\hline
\end{tabular}

\section{CONCLUSIONS}

Information and Communication Technology, have changed the role of the libraries in acquire, maintain, manage and distribute the information, to the end users. The main purpose of library automation is, to access the main modules and functions of the library. The library and professionals, allow them to contribute more meaningfully to spread the knowledge and Information. This paper highlights the full featured OSS and commercial software, of library automation like KOHA, LibSys, NewGenLib and SOUL. This study also focuses on the features, functions, modules and standards of the library automation software, and it highlights the design and architecture of specific software. Koha has more features than LibSys, NewGenLib and SOUL. The Koha is one of the open source technologies, and it is easy to install and easy to use. The NewGenLib has simple and useful software, for library automation. The commercial software like Libs' and SOUL are also available, but the Koha have more features than this commercial software. Overall, all the library automation software has advanced and one can select the Integrated Library Management System software, for their needs.

\section{REFERENCES}

1. Capterra. (2016). Top Library Automation Software Products. Retrieved 12-11-2016, from http://www.capterra.com/libraryautomation-software/

2. Engard, N. C. (2016). Koha 16.11: Koha 16.11 Manual (en). Retrieved 20-9-2016, from http://translate.kohacommunity.org/manual/master/en/pdf/Koha-16.11-koha-manual-en.pdf 
3. INFLIBNET. (2011). SOUL. Retrieved 20-9-2016, from inflibnet.ac.in/soul/

4. INFLIBNET. (2014). SOUL $2.0 \quad$ User Manual. Retrieved 20-9-2016, from http://www.inflibnet.ac.in/soul/downloads/Soul2.0\%20userManual.pdf

5. Joy, B. (2014). KOHA and LIBSYS: A Comparative Study, Journal of Advances in Library and Information Science, 3 (4), $350-$ 354

6. Koha-community. (2016). Koha Library Software: The world's first free and open source library system. Retrieved 12-11-2016, from https://koha-community.org/

7. LibSys. (2013). KibSys. Retrieved 20-9-2016, from http://libsys.co.in

8. LISWIKI. (2012). Criteria for evaluation of library software packages. 12-9-2016, from http://liswiki.org/wiki/Criteria_for_evaluation_of_library_software_packages

9. NCIP. (2016). NCIP Standing Committee. 20-8-2016, from www.ncip.info

10. Netugc. (2016). Library Management Software Packages. Retrieved 20-5-2016, from http://www.netugc.com/librarymanagement-software-packages

11. Rao, R. (1999). Features of Library Automation Software: A Comparative Study. SRELS Journal of Information Management, 36 (4), 211-228.

12. Reddy, C. S. V. (2013). Comparative Study of Free/Open Source Integrated Library Management Systems (FOSILMS) With Reference to Koha, Newgenlib and E-Granthalaya. e-Library Science Research Journal, 1 (12), 1-10.

13. Singh, K. (2012). NewGenLib: Open Source Software's In Indian Libraries. International journal of advanced research in management and social sciences, 1 (6), 173-179.

14. Verussolutions. (2016). NewGenLib. Retrieved 12-11-2016, from http://www.verussolutions.biz/web/

15. WordPress. (2009). NewGenLib" Open source library management software. Retrieved 12-11-2016, from https://newgenlib.wordpress.com 